Materiais e métodos: Foram recolhidos 80 incisivos de ovelha que foram divididos em dois grupos controlo: G1- controlo positivo; G2- controlo negativo, cada um com n=10; e em quatro grupos experimentais: G3- IRM ${ }^{\circledR}$; G4- KetacTM Silver (3M ESPE, Seefeld, Germany); G5- CavitTM (3M ESPE, Seefeld, Germany); G6- FiltekTM Supreme (3M ESPE, Seefeld, Germany); cada um com $n=15$. Os dentes foram seccionados, de modo a ficarem com $16 \mathrm{~mm}$ e foram desinfetados com cloreto de azidina. Realizaram-se as cavidades de acesso e procedeu-se à instrumentação canalar. Após aplicação dos materiais, os dentes foram submetidos a termociclagem (500 ciclos, $5 \pm 50 \mathrm{C} \mathrm{e}$ $55 \pm 5$ oC, por 30 segundos), impermeabilizados com verniz de unhas e selados apicalmente com cianoacrilato. Posteriormente à imersão em Pertecnetato de Sódio durante 3 horas, foi avaliada a infiltração através do processamento das imagens obtidas na gama-câmara. Foi utilizado o teste ANOVA para a análise estatística.

Resultados: Duas semanas após a aplicação dos materiais provisórios, observaram-se valores de microinfiltração em todos os dentes. Seguidamente à Resina Composta, o KetacTM Silver foi o que apresentou os menores valores de infiltração do radiofármaco, com significância estatística em relação aos restantes grupos. Pelo contrário, os dentes restaurados com CavitTM revelaram os valores mais elevados de infiltração. Quatro semanas após a aplicação do material provisório (T2), o compósito e o KetacTM Silver demonstraram valores de microinfiltração inferiores aos de T1 e aos restantes grupos. Também o CavitTM apresentou valores inferiores desde T1 a T2. Os valores mais elevados de microinfiltração passaram a pertencer ao IRM ${ }^{\circledR}$, sendo que este foi o único material que não apresentou diferenças estatisticamente significativas entre os diferentes tempos.

Conclusões: Após 2 e 4 semanas, o material provisório que apresentou menores valores de marcação com 99mTcNaO4 e, portanto, menor microinfiltração, foi o KetacTM Silver. Os valores mais elevados de microinfiltração foram encontrados às 2 semanas, nos dentes restaurados com CavitTM. No entanto, após 4 semanas os valores mais elevados de microinfiltração foram apresentados pelo grupo de dentes restaurados com IRM ${ }^{\circledR}$. http://doi.org/10.24873/j.rpemd.2017.12.127

\#105 Diferentes técnicas para Cimentação adesiva: comparação entre duas resinas compostas

Fátima Isabel Azevedo de Sousa*, Rui Isidro Falacho, Luís Roseiro, Francisco Caramelo, João Carlos Ramos, Fernando Guerra

Instituto Superior de Engenharia de Coimbra, Faculdade de Medicina da Universidade de Coimbra

Objetivos: Avaliar a espessura da interface restauração-agente cimentante-dente usando diferentes resinas compostas e diferentes técnicas na cimentação adesiva, com ou sem a aplicação de vibração ultrassónica e termo-modificação, evidenciando as diferenças e semelhanças relativamente à espessura de película.

Materiais e métodos: 72 discos confecionados em resina composta IPS Empress ${ }^{\circledR}$ Direct Ivoclar Vivadent foram aleato- riamente distribuídos por 6 grupos $(\mathrm{n}=12)$ e cimentados em pares utilizando: Variolink Esthetic LC Ivoclar Vivadent (Grupo 1), IPS Empress ${ }^{\circledR}$ Direct Ivoclar Vivadent termo-modificado (Grupo 2), IPS Empress ${ }^{\circledR}$ Direct Ivoclar Vivadent termo-modificado com vibração ultrassónica (Grupo 3), Estelite Omega ${ }^{\circledR}$ Tokuyama termo-modificado (Grupo 4), Estelite Omega ${ }^{\circledR}$ Tokuyama termo-modificado com vibração ultrassónica (Grupo 5) e Estelite Omega ${ }^{\circledR}$ Tokuyama à temperatura ambiente com vibração ultrassónica (Grupo 6). Para uniformização do protocolo experimental e dotá-lo de validade interna e externa, foi desenvolvido um sistema mecânico para exercer uma força controlada e constante de $30 \mathrm{~N}$, calibrada a partir de um operador real, sobre os discos a cimentar. Através de microscopia eletrónica foi possível observar a película da interface resina-cimento-resina e medir a sua espessura através do programa de obtenção de imagem do microscópio. Os valores obtidos foram submetidos a análise estatística utilizando o teste de Kruskal-Wallis com comparações múltiplas ajustadas entre os pares de grupos.

Resultados: As amostras do grupo 1 e 3 apresentam valores de espessura de película significativamente menores que os restantes grupos. O menor valor de espessura de interface foi verificado nas amostras do grupo 1 , com diferenças estatisticamente significativas com o grupo 2 e 4 . A maior espessura foi observada no grupo 2, apresentando diferenças estatisticamente significativas em relação ao grupo 3. Nos grupos em que as amostras foram cimentadas com Estelite Omega (grupo 4, grupo 5 e grupo 6), não se verificam diferenças estatisticamente significativas entre eles.

Conclusões: Considerando as limitações do presente estudo, Variolink Esthetic LC Ivoclar Vivadent e IPS Empress ${ }^{\circledR}$ Direct Ivoclar Vivadent termo-modificado com vibração ultrassónica proporcionaram espessuras das interfaces adesivas de cimentação mais finas que IPS Empress ${ }^{\circledR}$ Direct Ivoclar Vivadent termo-modificado sem vibração ultrassónica e Estelite Ome$\mathrm{ga}^{\circledR}$ Tokuyama independentemente da técnica. http://doi.org/10.24873/j.rpemd.2017.12.128

\#106 Influência de adesivos universais na reparação de cerâmica de dissilicato de lítio

Bruna Martins*, Pedro Melo e Moura, Luís Proença, Ana Mano Azul, Mário Polido

Instituto Superior de Ciências da Saúde Egas Moniz (ISCSEM), Caparica, Portugal., Centro de Investigação Interdisciplinar Egas Moniz (CiiEM); ISCSEM, Caparica, Portugal.

Objetivos: Avaliar a resistência adesiva à microtração ( $\mu$ TBS) entre uma cerâmica de dissilicato de lítio e um material reparador, utilizando diferentes adesivos universais e fazendo variar a aplicação prévia de um primer de silano.

Materiais e métodos: Dez blocos de cerâmica de dissilicato de lítio (IPS e.max ${ }^{\circledR}$ CAD, Ivoclar Vivadent) foram condicionados com ácido hidrofluorídrico e aleatoriamente divididos em cinco grupos, de acordo com o protocolo de reparação a aplicar: G1(SiA): Primer de silano Bis-SilaneTM (Bisco) e adesivo AdperTM ScotchbondTM MultiPurpose (3MESPE); G2(SU): Adesivo ScotchbondTM Universal (3MESPE); G3(SiSU): Bis-SilaneTM 
e ScotchbondTM Universal; G4(F): Adesivo Futurabond U (VOCO); e G5(SiF): Bis-SilaneTM e Futurabond U. As amostras foram depois reparadas com uma resina nanohíbrida (Grandio $\left.{ }^{\circledR}, \mathrm{VOCO}\right)$. Após armazenamento em água desionizada a $370 \mathrm{C}$, foram obtidos palitos com $1 \mathrm{~mm} 2$ de área seccional e avaliou-se a resistência à microtração ( $\mu \mathrm{TBS}$ ) às 24 horas e 3 meses. O modo de falha foi analisado numa lupa estereoscópica (16x). A análise estatística foi efetuada com recurso aos testes t de Student, ANOVA One-way, Qui-quadrado e Fisher, para um nível de significância de 5\%.

Resultados: Os valores de $\mu$ TBS foram mais elevados na avaliação imediata: $\operatorname{SiA}(36.83 \pm 15.22 \mathrm{MPa})>\operatorname{SiF}(31.70 \pm 10.12 \mathrm{MPa})>$ SiSU $(30.37 \pm 8.66 \mathrm{MPa})>\mathrm{SU}(23.51 \pm 8.86 \mathrm{MPa})>\mathrm{F}(22.30 \pm 7.87 \mathrm{MPa})$. Observaram-se diferenças significativas entre o grupo SiA e o grupo SU e F e entre os grupos SiF e F ( $p=.001)$. Após armazenamento durante 3 meses ocorreu falha pré-teste nos grupos SU e F, e uma diminuição significativa nos valores de $\mu$ TBS dos restantes grupos: SiA $(26.21 \pm 6.43 \mathrm{MPa}) \quad(\mathrm{p}=.003)>\mathrm{SiF}$ $(24.14 \pm 8.27 \mathrm{MPa})(\mathrm{p}=.013)>\operatorname{SiSU}(22.20 \pm 6.06 \mathrm{MPa})(\mathrm{p}=.001)$. No que concerne ao modo de falha, não houve diferença estatisticamente significativa entre os grupos de avaliação às $24 \mathrm{~h}(\mathrm{p}=.072)$ contrariamente ao ocorrido após envelhecimento $(\mathrm{p}=.006)$.

Conclusões: A $\mu$ TBS entre um material restaurador e uma cerâmica de dissilicato de lítio varia de acordo com a aplicação, ou não, de um primer de silano previamente ao adesivo universal, assim como com o tempo de armazenamento. A reparação de restaurações em cerâmica de dissilicato de lítio CAD/ CAM com a utilização de um primer de silano seguido da utilização de um adesivo universal é preferível à utilização de um adesivo universal isolado.

http://doi.org/10.24873/j.rpemd.2017.12.129

\section{\#107 Anatomia canalar do primeiro molar inferior definitivo - Revisão sistemática ilustrada}

João Meirinhos*, Mariana Peixe Domingos Alves Pires, Isabel Beleza de Vasconcelos, Jorge Martins, Mário Rito Pereira, António Ginjeira

Faculdade de Medicina Dentária da Universidade de Lisboa

Objetivos: O sucesso do tratamento endodôntico depende da desinfeção do sistema de canais radiculares. O conhecimento da anatomia mais comum desses canais, assim como as suas variações, torna-se fundamental para que os objetivos dessa mesma desinfeção sejam alcançados. Diversas metodologias têm vindo a ser empregues para o estudo da anatomia do sistema de canais radiculares, que vão desde radiografias, resultados clínicos, diafanização e, mais recentemente, técnicas tridimensionais como o tomografia computadorizada de feixe cónico (CBCT) ou a micro tomografia computorizada (micro-CT). Tendo em conta que o primeiro molar inferior é o dente mais submetido a tratamento endodôntico, torna-se ainda mais importante o seu conhecimento anatómico interno. O objetivo principal deste estudo é analisar a configuração mais comum, e possíveis variações, da anatomia canalar do primeiro molar inferior segundo a nomenclatura descrita por Vertucci (1984). Para isso foi revista, de um modo sistemático, a literatura cujos estudos de anatomia recorressem a técnicas de diafanização, CBCT ou micro-CT. Como objetivo secundário, procurou-se realizar a ilustração de algumas configurações anatómicas identificadas, recorrendo à reconstrução tridimensional de amostras analisadas em micro-CT.

Materiais e métodos: Foi efetuada uma pesquisa nos motores de busca de duas bases de dados primárias (PubMed e ScienceDirect), em Julho de 2017, com a seguinte conjugação de termos: (molar) AND (Vertucci) AND (CBCT OR clearing OR micro-CT), sem filtros ou restrição de linguagem, para artigos publicados a partir de 1974. Os títulos e resumos dos trabalhos identificados foram revistos e selecionados os estudos pertinentes. Para esses foram lidos os manuscritos e feita a revisão das referências bibliográficas em busca de novos trabalhos.

Resultados: Foram incluídos 20 estudos resultando numa amostra de 4824 primeiros molares inferiores permanentes. Os tipos de anatomia canalar mais frequentes foram os tipos IV $(59,1 \%)$, II $(23,8 \%)$ e I $(17,6 \%)$ para a raiz mesial, e tipos I (54,8\%), II (19,2\%) e IV (11,6\%) para a raiz distal.

Conclusões: Estudos sobre a anatomia canalar recorrendo a técnicas 3D são capazes de fornecer informações importantes sobre a morfologia e configuração interna do sistema de canais. Quando abordando endodonticamente o primeiro molar inferior, o clínico pode esperar alguma variabilidade anatómica em ambas as raízes.

http://doi.org/10.24873/j.rpemd.2017.12.130

\section{\#108 Biocompatibilidade de Novos Cimentos} Endodônticos

Joana Freitas, Diana Sequeira*, Paulo Jorge Rocha Palma, Ana Luísa Cardoso, João Peça, João Miguel dos Santos

Faculdade de Medicina da Universidade de Coimbra, Centro de Neurociências e Biologia Molecular da Universidade de Coimbra

Objetivos: Este estudo tem como objetivo principal avaliar a biocompatibilidade de um novo cimento endodôntico à base

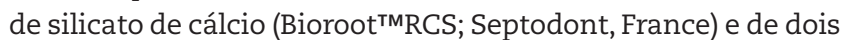
novos cimentos à base de silicone (Guttaflow ${ }^{\circledR} 2$ e GuttaFlow ${ }^{\circledR}$ Bioseal; Coltène Whaledent, GmBH Co KG, Langenau, Switzerland). Neste projeto foi usado como referência um cimento gold standard, largamente utilizado na clínica e em estudos prévios, à base de resina epóxi (AH Plus ${ }^{\circledR}$, Maillefer Dentsply, Ballaigues, Switzerland). O objetivo secundário foi testar a solubilidade destes cimentos de obturação canalar após imersão em meio de cultura durante $24 \mathrm{~h}$.

Materiais e métodos: As células do ligamento periodontal foram incubadas com eluatos dos quatro cimentos à temperatura de $37^{\circ} \mathrm{C}$ numa atmosfera humedecida contendo $5 \%$ de $\mathrm{CO} 2$ durante $24 \mathrm{~h}, 48 \mathrm{~h}$ e $72 \mathrm{~h}$. Foram testadas diferentes concentrações ao longo do tempo de modo a determinar qual a dose-resposta e a exposição-resposta das células a estes materiais. A citotoxicidade foi determinada através do teste Alamar Blue ${ }^{\circledR}$ e confirmada com microscopia electrónica. A análise estatística foi efetuada recorrendo ao programa Prism (GraphPad Software, CA). Foram aplicados os testes Kolmogorov-Smirnov; Two-way ANOVA com Tukey post hoc; One-way ANOVA com Dunnetts post hoc; e T-test. Para valor 